layers, and beyond, remain unanswered. Structural probes that analyze cluster formation do not address the important issue of the movement of water on surfaces."

\section{Copper Found in Bloodworm May Serve as Paradigm for New Materials}

Researchers report in the October 11 issue of Science their detection of a copperbased biomineral in the jaw tip of Glycera dibranchiata, better known as the marine bloodworm, the bait of choice for certain kinds of fish. This is in contrast to the much more commonly observed Ca-, Si-, or Fe-based minerals. Herbert Waite of the University of California-Santa Barbara (UCSB) and colleagues determined that copper also occurs in nonmineral form in the bloodworm jaw where it may act as a structural element in cross-linking long chains of fibrous proteins. The researchers reported, "The marriage of protein with copper mineral as well as with bound copper ions is an intriguing concept per se but may also serve as a design prototype for new materials that need to be hard, lightweight, and durable."

In addition, the research team found the non-mineral form of copper on the surface of the jaw canal through which venom is injected. That copper may be acting as a catalyst that activates venom being discharged by the worm into its prey, they said.

The researchers found that the jaw tip exhibits the ordered crystalline structure of the copper-based biomineral atacamite $\left[\mathrm{Cu}_{2}(\mathrm{OH})_{3} \mathrm{Cl}\right]$. They found that the bloodworm's unmineralized copper as well as the copper biomineral is concentrated in the first half millimeter of the jaw, and that the copper biomineral is organized in nanostructured fibers $(\sim 50 \mathrm{~nm}$ in diameter) within a protein matrix. They reported that the "fiber orientation within the tip is roughly parallel to its outer shape."

Through nanoindentation, performed by Thomas Schöberl of the Erich Schmid Institute for Materials Science, Leoben, Austria, the researchers mapped hardness $(H)$ and stiffness $(E)$ with composition in the bloodworm jaw. They then determined that both hardness and stiffness increased with increasing mineral content. The hardness-to-stiffness ratio in bloodworm jaws was higher than in other known mineralized tissue. According to the research team, this was indicative of a high abrasion resistance, commonly understood as equal to hardness to the three halves power divided by stiffness $\left[H^{3 / 2} / E\right]$.

"With the worm," said Waite, "it is that quotient that is revealing rather than individual hardness and stiffness meas- urements. So when you look at that quotient, the wear resistance value is very close to the best materials we can make."

But high values for $H$ and $E$ also correlate with the presence of copper, even where the copper is not in mineral form. First author Helga Lichtenegger's analysis of the distribution of copper in the bloodworm jaw suggested that about half the copper was in a biomineral form and half in the form of copper aggregates.

Waite said, "I know from my research that the proteins in invertebrate jaws and particularly in the jaw tips are not complicated. They have generally just two types of amino acids, glycine and histidine. These two amino acids probably repeat in a precise chainlike sequence. In this respect it is one of nature's closest analogues to synthetic polymer molecules. I expect that the copper cation in the bloodworm jaw plays a role in the cross-linking of these amino acid chains."

"Storing venom in nontoxic form that is being catalytically activated as it passes through this syringe may," suggested
Waite, "afford us a model for delivering an unstable chemical by activating a stable form of it as part of the parting shot." Among the applications he envisions is a system for delivery of unstable drugsthose with a short shelf life. "We may be able to synthesize the drug in a precursor form and have that last important step done as part of delivery."

\section{FOR MORE RESEARCII NEWS ON MAIERLALS SCLENCE ... \\ ... access the Materials Research Society Web site: \\ www.mrs.org/gateway/ matl_news.html}

\section{MRS Spring Meeting April 21-25 - San Francisco, CA \\ See page 999 for more information}

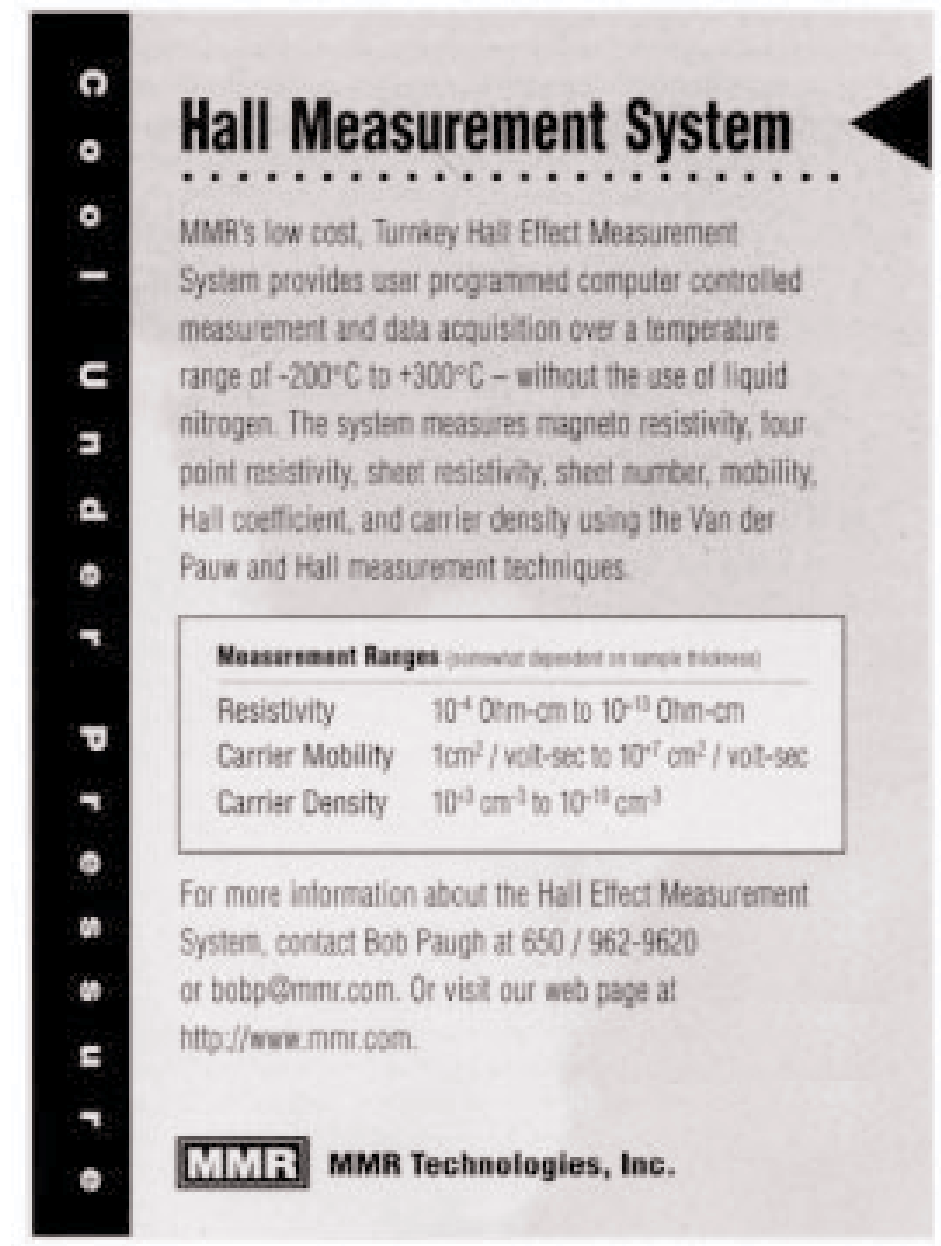

Circle No. 12 on Inside Back Cover 